Classificątion

Physics Abstracts

$07.80-82.80-87.80-06.50$

\title{
Electron Spectroscopic Imaging of Organic Compounds Using PC- Based Energy Sequence Imaging Software
}

\author{
Andrew D. Johnson $\left({ }^{1}\right)$, Robert J. Mairs $\left({ }^{2}\right)$, Mark N. Gaze $\left({ }^{3}\right)$, Gretel Sass $\left({ }^{4}\right)$ and Ian M. \\ Huxham $\left(^{1}\right)$ \\ $\left({ }^{1}\right)$ Molecular \& Cellular Biology Division, EM Centre Joseph Black Building; University of Glasgow, \\ Glasgow, G12 8QQ, UK \\ ( $\left.{ }^{2}\right)$ Department of Radiation Oncology, University of Glasgow, Glasgow G12 8QQ, UK \\ $\left({ }^{3}\right)$ Meyerstein Institute of Clinical Oncology, The Middlesex Hospital Medical School, London W11 \\ 8AA, UK \\ $\left({ }^{4}\right)$ medac GmbH, Fehlandstrasse 3, D-20345 Hamburg, Germany
}

(Received October 7, 1994; accepted February 8, 1995)

\begin{abstract}
We have designed, characterised and tested Windows based software packages for rapid elemental microanalysis using a sequence of energy filtered transmission electron microscopic images which combines electron spectroscopic imaging with electron energy loss spectroscopy (Image-EELS). Image sequences containing up to 45 images of $512 \times 512$ pixels were recorded rapidly over an appropriate range of the energy spectrum for an analysis of boron, nitrogen, titanium and fluorine. Energy loss information was extracted from local variations in the energy loss spectrum with good energy resolution $(5 \mathrm{eV})$ and a spatial resolution of $2 \mathrm{~nm}$ for the determination of the relative concentration of elements within a region of interest by grey level integration in consecutive images of an energy loss sequence, using the least mean squares method to calculate the parameters for an $A \cdot e^{-r}$ background model. The methods were used to define the optimum collections conditions and practical limitations of the system for qualitative and semi-quantitative elemental analysis relevant to biological specimens. Qualitative elemental analysis of images from Lowicryl HM23- embedded ultra-thin section of a boric acid polymer showed that boron was not distributed evenly. Semi-quantitative elemental analysis of the fluorine-containing organic polymer Nafion suggested a linear relationship between fluorine energy loss intensity, image area and specimen thickness. These techniques were applied to an examination of the reaction between purified RNA and the novel titanium-containing anti-cancer agent MKT-4, showing that ratios calculated from the cumulative electron energy loss intensity increase within regions of interest as a function of grey level intensity for titanium and nitrogen are directly proportional to the incubation concentration of the drug with RNA. Expression of data in this way is shown to be a useful indicator of the relative drug distribution within specific intracellular compartments of human cancer cells incubated with MKT-4 in vitro.
\end{abstract}




\section{Introduction}

One experimental approach to energy filtering electron microscopy combines electron spectroscopic imaging with electron energy loss spectroscopy. The technique, known as Spectrum Imaging [1], Image Spectrum [2] or Image-EELS [3], records energy specific elemental loses as a grey scale within an image array from one or more elements within a large area for several images over a defined electron energy loss range. In addition to elemental information, the digital image sequence also contains grey level intensity variations corresponding to information about local changes in mass/thickness and the non-specific background energy contributions, which can be determined by modelling the local energy spectrum. This energy filtered image sequence approach allows the rapid, correlative microanalysis of several elements within defined regions with the best possible sensitivity, and is particularly useful for an accurate assessment of native and experimental compounds present as local concentrations of diffuse constituents, as found in biological cells $\&$ tissues, which do not form electron-dense precipitates.

Our aims were to create a flexible Windows based program which could be used on any PC fitted with a video board in order to permit a preliminary pixel-by-pixel analysis of energy loss in electron spectroscopic $512 \times 512$ pixel image sequences, for the whole image or for regions of interest. Secondly, to test the practical application of the modelling algorithm to image sequences by refining the summed spectrum concept of Jeanguillaume and Colliex [4] following generation of element distribution images (elemental maps) of boron in a Lowicryl- embeded boric acid poly$\operatorname{mer}(\Delta E=150-250 \mathrm{eV})$ and also for semi-quantitative analysis of fluorine $(\Delta E=650-750 \mathrm{eV})$ in thin films of the polymer Nafion. These two carbon based polymers were used as model systems to determine the optimum conditions for the collection of image sequences of trace elements in biological material within an organic matrix. As an application of the technique, we examine purified RNA incubated with increasing concentrations of the cytotoxic anti-cancer agent titanocene dichloride [5, 6] and in human ovarian cell spheroids following incubation with the drug in vitro in order to examine the qualitative and semi-quantitative intracellular distribution of the drug by determining the relative titaniun/nitrogen energy loss intensity ratios.

\section{Polymer Preparation}

Boric acid gel (Aldrich) was swelled in ethanol and embedded in Lowicryl HM23 methylacrylate resin at $-20^{\circ} \mathrm{C}$, and polymerised using UV light. This resulted in an amorphous mixture containing electron-dense boron-rich domains and less dense support resin domains. Semi thin sections with a nominal thickness of $50-60 \mathrm{~nm}$ were cut using a glass knife and mounted unsupported onto 700 mesh gold grids. A zero-loss bright field image of the boric acid polymer (BAP) is shown in Figure 1. Nafion EW1100 (Sigma, Poole Dorset, UK; containing 70\% + / - 1\% fluorine and $22 \%+/-1 \%$ carbon by weight) thin films were prepared by spreading $3 \mu \mathrm{l}$ of a $0.7 \%$ polymer solution dissolved in an ethanol/water mixture (95\%/5\% volume/volume) onto a mica surface. This method resulted in a thin film of polymer of constant thickness (measured in cross section) in which fluorine was distributed evenly. Evaporated thin films with nominal thickness of $60 \mathrm{~nm}$ were floated and lifted onto uncoated grids and desiccated before analysis. A zero-loss brightfield image of Nafion is shown in Figure 2. 


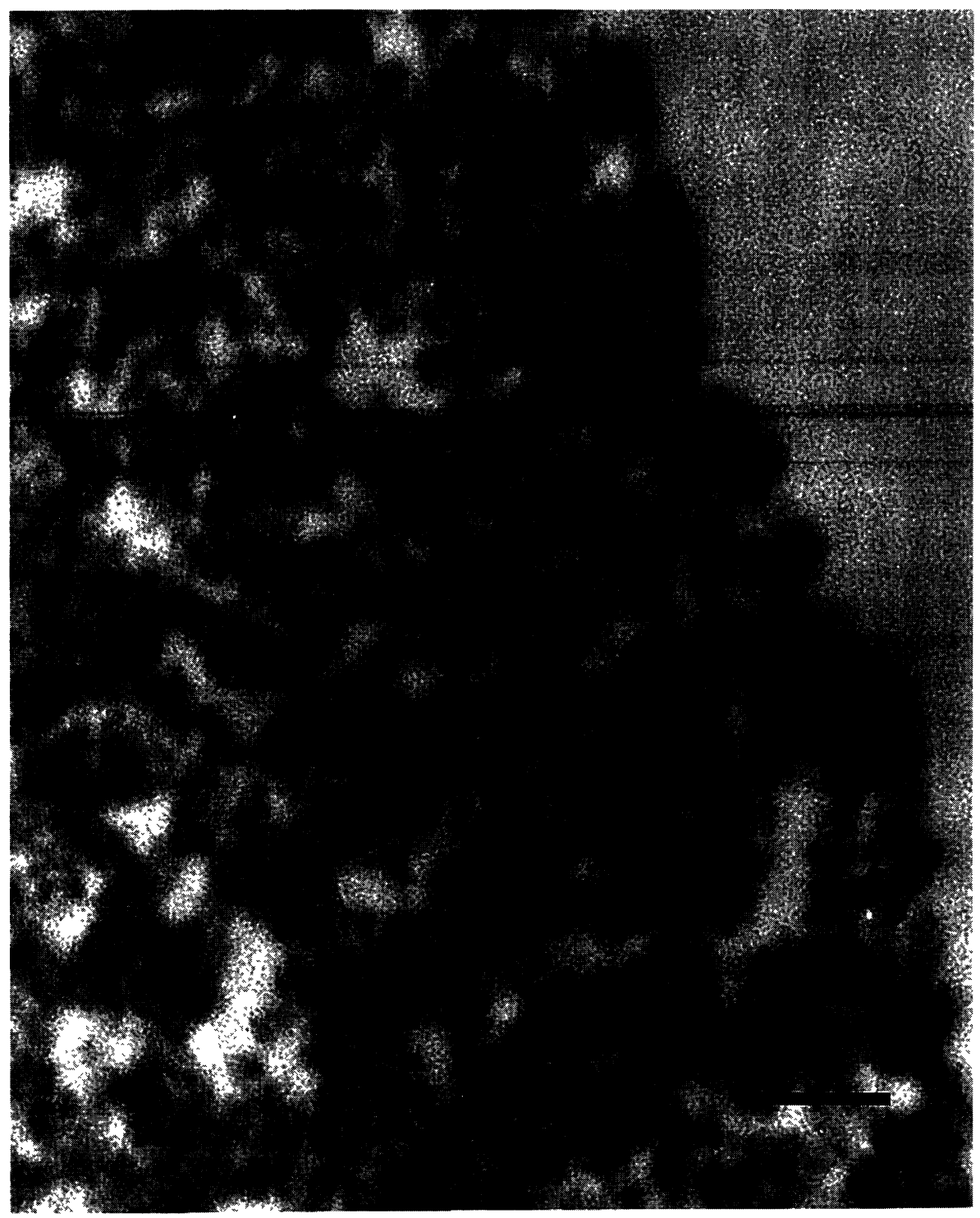

Fig. 1. - Zero-loss energy filtered bright field micrograph of the native boronic acid polymer embedded in HM23 resin. The darker, more electron dense regions represent local domains of the polymer and the lighter regions primarily resin. $\mathrm{Bar}=400 \mathrm{~nm}$.

\section{Titanocene Dichloride Incubation with RNA and with Humann Ovarian A2780 Multicellular Spheroids}

Titanocene dichloride formulations (MKT-4, mol mass 249) were supplied by medac (Hamburg, Germany), dissolved in saline to a concentration of $5 \mathrm{mg} / \mathrm{ml}$ and used immediately. RNA (Product number R7250, Sigma, Poole UK, chosen because it is a major component of cells, can be obtained cheaply in a pure form, and is highly resistant to both chemical insult and electron beam 


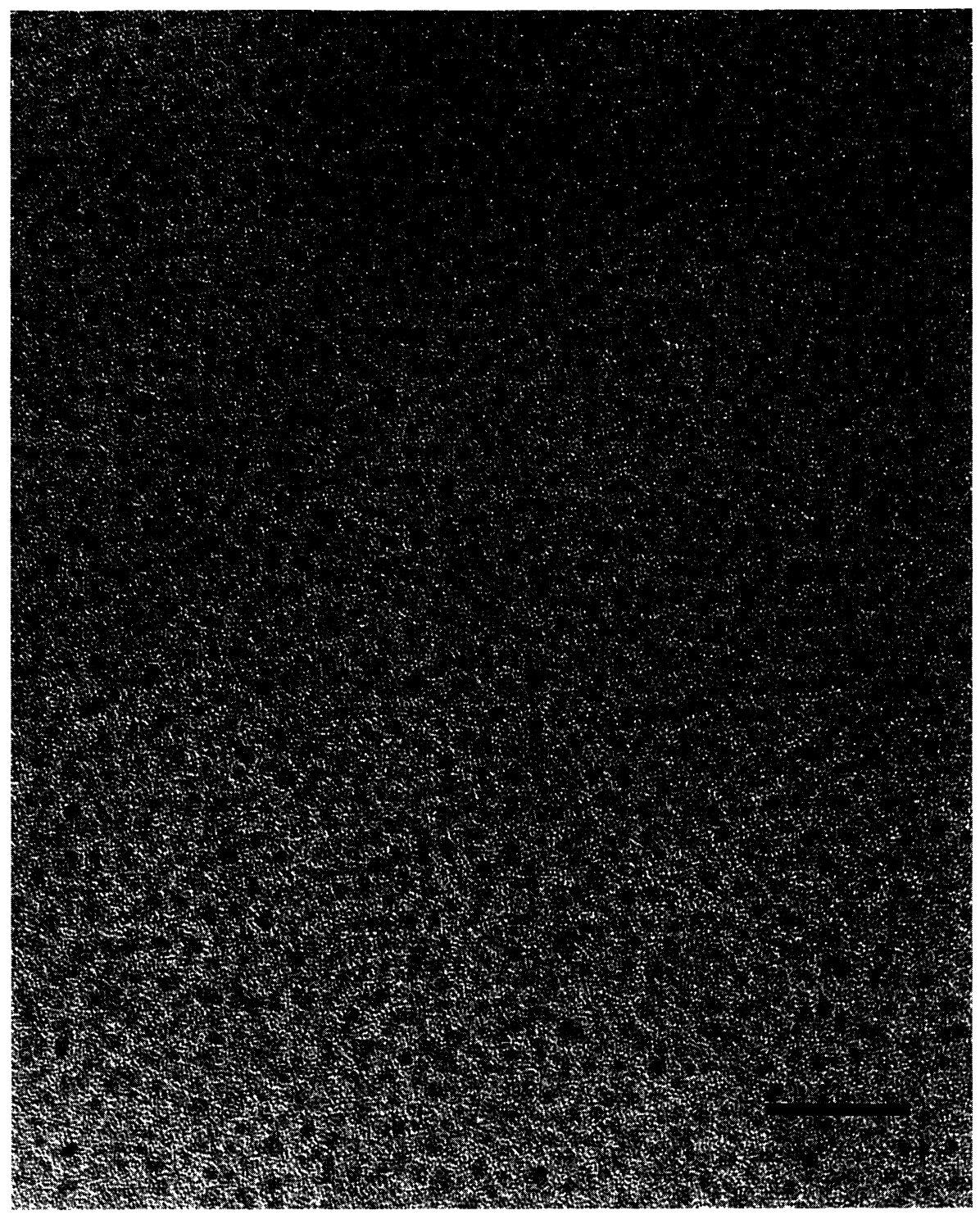

Fig. 2. - Zero-loss energy filtered bright field micrograph of Nafion (tetrafluoroethylene and perfluorovinyl ether 7:1). Although Nafion is not cross linked, the hydrophophilic sulphonate groups arrange during preparation to form clusters (sulphonate domains) and act as transient cross links which contribute to polymer rigidity. $\mathrm{Bar}=50 \mathrm{~nm}$.

irradiation) was dissolved in saline to a concentration of $30 \mathrm{mg} / \mathrm{ml}$ and incubated in a total volume of $60 \mu \mathrm{l}$ with $250,125,62.5,31$ and $15.5 \mu \mathrm{g}(16,8,4,2$ and $1 \mathrm{mM})$ of MKT-4 for 5 min at room temperature. RNA-MKT-4 conjugates were washed three times by precipitation using diltute hydrochloric acid ( $\mathrm{pH} \mathrm{2.0)}$ and centrifugation, then re-dissolved in water before spread mounting and air drying on silicon oxide support films (Agar Scientific, $\mathrm{UK}, t / \lambda=0.15$ ). The aim was to determine whether or not the drug would bind to RNA in a concentration-dependent manner. 
The conjugate could also facilitate the interpretation of energy loss image sequences following any differential drug binding to the intracellular compartments of cancer cells in vitro by allowing a comparison between the titanium/nitrogen energy loss intensity ratios within specific cellular compartments and the conjugate to be made. Intensity ratios accommodate any MKT-4-bound RNA density differences in the spread preparation by normalisation of the data to the magnitude of the intensity increase associated with nitrogen content. A zero-loss brightfield image of the conjugate is shown in Figure 3.

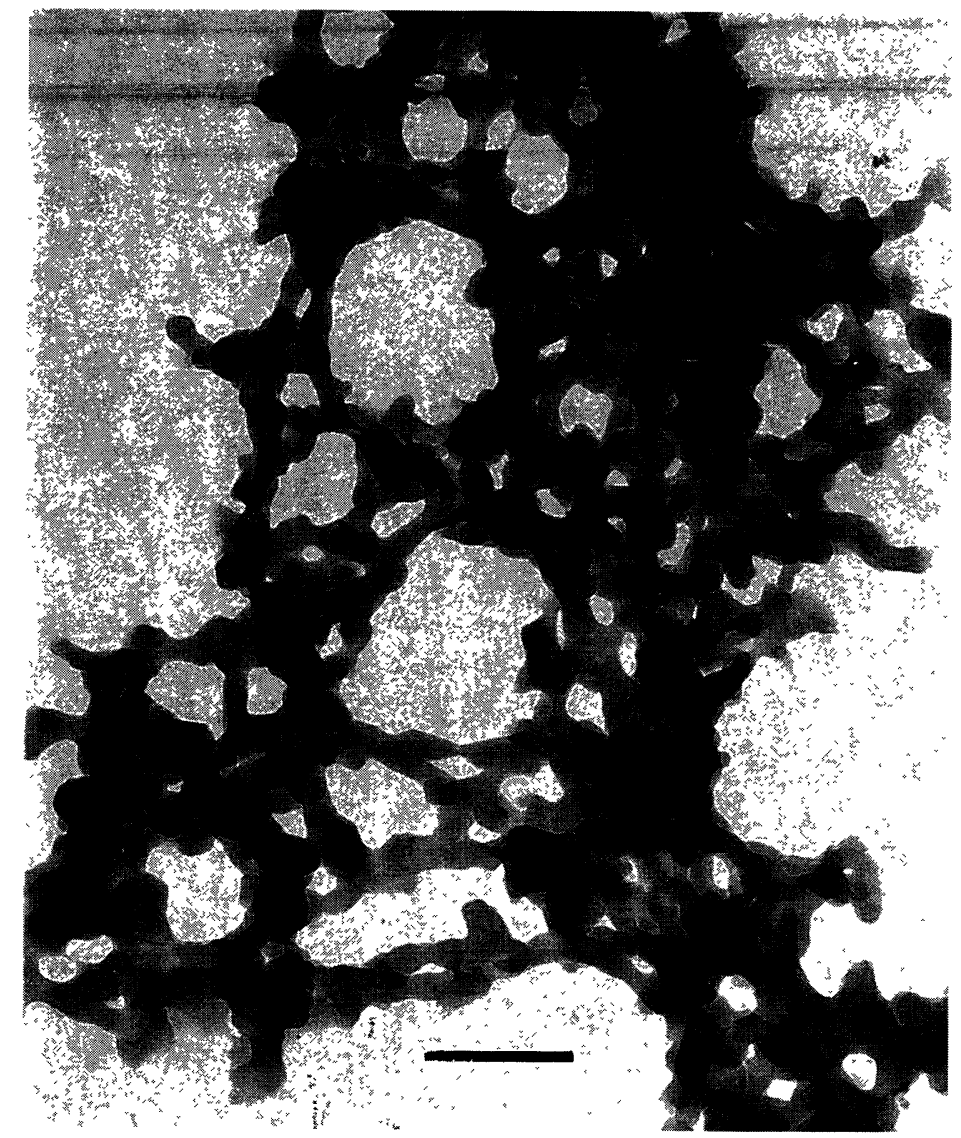

Fig. 3. - Brightfield image of the RNA-MKT-4 conjugate after air-drying onto a silicon monoxide support film. Bar $=200 \mathrm{~nm}$.

Human ovarian A2780 cancer cell multicellular spheroids were prepared as described elsewhere [7] and cultured with $500 \mathrm{uM} \mathrm{MKT-4}$ for $24 \mathrm{hrs}$ in $\mathrm{CO}_{2}$-independent medium (Gibco, Paisley, Scotland) at $37^{\circ} \mathrm{C}$. Spheroids were washed in drug-free medium, then in ice cold phosphate buffered saline, and chemically fixed with phosphate buffered $1 \%$ glutaraldehyde on ice for $1 \mathrm{hr}$. Following low-temperature dehydration and embedding in nitrogen-free Lowicryl HM23 resin [8], ultra-thin sections of cells on the outer edge of the spheroid were examined unstained as above for the RNA-MKT-4 conjugate. 


\section{Electron Energy Loss Spectroscopy}

The Zeiss TEM 902 operating at $80 \mathrm{kV}$ was set up for serial electron energy loss spectroscopy (SEELS) and electron energy loss image sequence recording (image-EELS) using the $400 \mu \mathrm{m}$ condenser aperture, the $90 \mu \mathrm{m}$ objective aperture (22 mrad), with the $100 \mu \mathrm{m}$ spectrometer entrance aperture for SEELS and the $400 \mu \mathrm{m}$ entrance aperture for Image-EELS, a focused beam current of $125 \times 10^{-8}$ amp in a vacuum of $7 \times 10^{-7}$ Torr at room temperature. Use of a high beam current was adopted to maximise the signal/noise ratio. An intermediate aperture $(50 \mu \mathrm{m})$ was inserted to select an area of $1.5 \mu \mathrm{m}^{2}$ for analysis at 20,000 times magnification.

Relative thickness was determined by serial EELS (SEELS) via an A/D converter (PCL-812 Lab Card) with up to 300 data points per $6 \mathrm{eV}$, with an energy window (spectrometer exit slit width) of $6 \mathrm{eV}$ in order to match the dimensions of the photo-multiplier tube (PMT) detector window. This set-up was found to be the only way to maintain reproducibility of the SEELS signal intensity from one region to another. An intensity value of 1000 on the serial plot corresponded to a recorded current of $1 \mu \mathrm{A}$ from the PMT. Relative thickness $(t / \lambda)$ was calculated from the formula $t=$ $(\lambda) \ln \left(I_{\mathrm{t}} / I_{\mathrm{o}}\right)$ as described previously [9]. SEELS measurements were made immediately after image sequence collection on the same area of the specimen.

For Image-EELS, a $5 \mathrm{eV}$ exit slit was used to collect 15-45 consecutive images (focused at $\Delta E=250 \mathrm{eV}$ ) with $5 \mathrm{eV}$ increments over $78 \mathrm{~s}$ from a Dage SIT camera via a fluorescent screen, each integrated 20 times to improve the signal/noise ratio, using a DT-2861 video board with 16 buffers located within a 486 PC. Image sequences containing an array of $512 \times 512$ pixels with $0-255$ grey level values were stored on an optical disc for off-line analysis. Since the camera response to light is known to be essentially linear and the dark current contributuion is practically negligable [10], simple addition of the recorded grey level intensity values as a function of energy loss for sequential images was adopted as a reasonable representation of energy loss. Sequences in which significant specimen drift occurred were discarded. Image sequences of BAP were compared directly with sequences of resin immediately adjacent to the polymer ( $<1 \mu \mathrm{m}$ distance) at 20,000 times magnification ( 1 pixel $=3.7 \mathrm{~nm}$ ), without changing any of the microscope settings or camera controls. Image sequences for the BAP were collected as rapidly as possible and were matched so that $79-86 \%$ of the area for analysis was occupied by boron-rich domains. Image sequences were similarly recorded from Nafion at 20,000 times magnification and from resin with similar $t / \lambda$ values to Nafion for comparative analysis retrospectively, again without changing any of the instrument or camera settings. Grey level profiles were recorded across the centre of an image using PC_IMAGE Plus package (Foster Findlay Associates, Newcastle. UK). All images were modelled without any shading correction or dark current correction, but were median filtered to reduce 'noise'. The effects of instrument aberration on the local variation of ionisation edge onset within an image sequence were investigated using 45-image sequences of Nafion recorded using a $1 \mathrm{eV}, 2.5 \mathrm{eV}$ and $5 \mathrm{eV}$ spectrometer exit apertures as above. Energy filtered image sequences containing 45 images of selected areas of the RNA-MKT-4 conjugates were recorded at 50,000 times magnification ( 1 pixel $=1.5 \mathrm{~nm}$ ) between $\Delta E=350-570 \mathrm{eV}$ using a $5 \mathrm{eV}$ window as described above, in order to record both the nitrogen K-edge and titanium $L_{2,3}$ energy loss intensity information in each sequence

\section{Background Modelling}

Every pixel in a spectroscopic image sequence $(512 \times 512 \times 45)$ was stripped of its background intensity contribution by approximation to an $A \cdot e^{-r}$ curve, where the constants $A$ and $r$ were determined by using a least squares method [11] rather than the previously adopted two and three window methods, in order to define the immediate pre-edge shape of the energy spectrum 
more precisely for accurate projection beyond the ionisation event. In all cases, the modelled background contribution of each $5 \mathrm{eV}$ image or regions of interest (ROI) within an image was subtracted from the recorded value and the result displayed as a summed intensity of the relative energy loss. Our method differs from both the Imaging-spectrum approach [2] and Kortje's Image-EELS [3] approaches in that it sums rather than averages all grey level intensity values within a ROI in order to maximise sensitivity for visual display of the energy spectrum or for deviation of the stripped intensity value spectrum. In addition our background modelling calculations for $A$ and $r$ were derived from the original individual grey level pixel values in the image sequence and not from values in the displayed spectrum.

\section{Analysis of BAP}

The signal intensity for boron decreased rapidly following three minutes of beam exposure without any appreciable decrease in $t / \lambda$, suggesting preferential mass loss of boron by radiation damage. SEELS analysis of BAP (Fig. 4) using the intermediate aperture to select an area of $1.5 \mu \mathrm{m}^{2}$ demonstrated a good boron ionisation event. Using both our hardware-dependent and independent Imaging software it was possible to measure the boron- specific intensity increase associated with a single pixel $(3.7 \mathrm{~nm})$ within polymer-rich domains. In this region of the spectrum, most pre-edge stripped images contained a grey-level value variation of between 6 and 14, so a threshold intensity value of 135 was used to define significant energy loss in these image sequences. Values for the resin only $(t / \lambda=0.198)$ region were fairly high, constituting $25 \%$ of the values recorded for BAP-containing regions. Cumulative grey-level intensity values per pixel for square

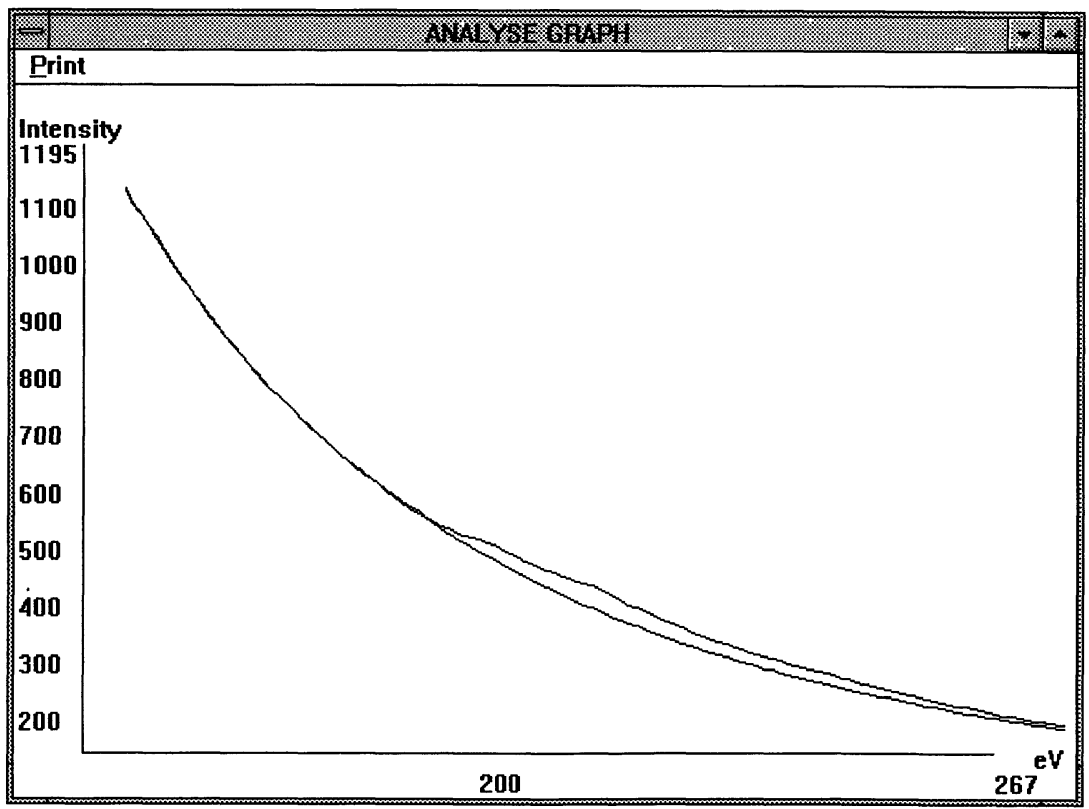

Fig. 4. - Serial electron energy loss spectrum of the boronic acid polymer. The boron K-edge (edge onset at $\Delta E=188 \mathrm{eV}$ ) is detectable as the upper line. The lower line represents the projected background intensity values determined by the least mean squares method on the basis of the recorded values between $\Delta E=150-188 \mathrm{eV}$. 
ROI's centred at point $x 256, y 256$ on the image calculated from 5 post-edge images $(190-215 \mathrm{eV}$, $t / \lambda=0.229$ ) were close to linear; ROI's comprising $2500,10,000,22,500,40,000$ and 126,888 pixels were $800,650,600,550250$ for BAP and 320, 133, 125, 51 \& 39 for resin respectively. An increase in section relative thickness within the range 0.2 to 0.3 was associated with a marked and corresponding increase in the cumulative post-edge grey level value for all ROI's, but this increase was only linear for ROI's with $<=50,000$ pixels. Although the grey level intensity increase associated with energy loss for boron in the polymer with $t / \lambda<=0.3$ was significant (around 20 for a single pixel of $3.7 \mathrm{~nm}$ using a $5 \mathrm{eV}$ energy window), we were unable to define any precise relationship between the intensity grey level increase and the mass proportion of boron in this polymer because of local variations in polymer density coupled to large variations in the background contributions of neighbouring pixels. Despite these limitations, we find that averaging the background-stripped boron post-edge images would be the best method for determining the qualitative distribution of boron in the polymer, but that summing the same post-edge images is likely to provide both a similar boron distribution image together with an indication of the relative changes in local boron concentration within specific domains, as shown by a much greater local change in the dynamic range of the resultant image. However, semi-quantitative analysis of boron in this sort of image would be difficult, particularly for large areas, because of the high variation accross the image in background contributions relative to a comparatively weak boron-associated image intensity increase.

\section{Analysis of Nafion}

The polymer was surprisingly resistant to a high beam current and could survive intact without detectable signal intensity loss for up to $2 \mathrm{~min}$ of irradiation. No change in the relative thickness of these films was detected before this time. The overall grey-level intensity decrease in this range of the energy spectrum for both the polymer and the resin was significantly less than for BAP. Stripping each image using the hardware-dependent DT software by subtracting the modelled background contribution, assigned a grey level value of 128 for pixels in which no difference between the modelled value and the actual value was found and resulted in values greater than 128 for any positive values (i.e., $+1=129,+8=135,+20=149$, etc.). A grey level difference of almost 80 for a single pixel $(3.7 \mathrm{~nm})$ at $700 \mathrm{eV}$ using a $5 \mathrm{eV}$ energy window was common in the stripped images, whilst values of less than 5 were recorded for the resin with similar $t / \lambda$ (data not shown). The system noise for this region of the spectrum varied from 8 to 19 for pre-edge images, so a threshold grey level value of 145 was chosen for calculations of cumulative grey level values for the whole image. The intensity values associated with fluorine from 9 summed stripped images for a range of ROI increased linearly with area (for example, ROI; 2500, 10000, 22500, 40000, 62500 and 126888 pixels was associated with summed intensity values of $880,889,887,876$, 854 and 804 per pixel for Nafion and 4, 4, 4, 4, 4, and 3 per pixel for resin respectively), but this relationship was less robust for the larger sized ROI's ( $>=100000$ pixels), particularly for thinner (30 - $40 \mathrm{~nm}$ thickness) Nafion films. Unlike BAP, the background intensity contribution in this region of the energy spectrum derived form stripped images from material with a similar $t / \lambda$ without fluorine was negligible. Figure 5 shows a typical pixel-by-pixel stripped image sequence of Nafion modelled on original images $1-7(660-690 \mathrm{eV})$, where the top left hand side image is $660 \mathrm{eV}$, and the bottom right hand side image is at $735 \mathrm{eV}$, in which the edge onset for fluorine is clearly seen in frame 8 . An image of the intermediate aperture is seen in each frame. On the basis of the elemental composition of Nafion comprising $70 \%( \pm 1 \%$,) fluorine, we calculate that for our specific experimental conditions (i.e. summing the total fluorine energy loss intensity values from several post-edge stripped images), a grey level intensity increase of 1 corresponds to the presence of about 500 fluorine atoms. 


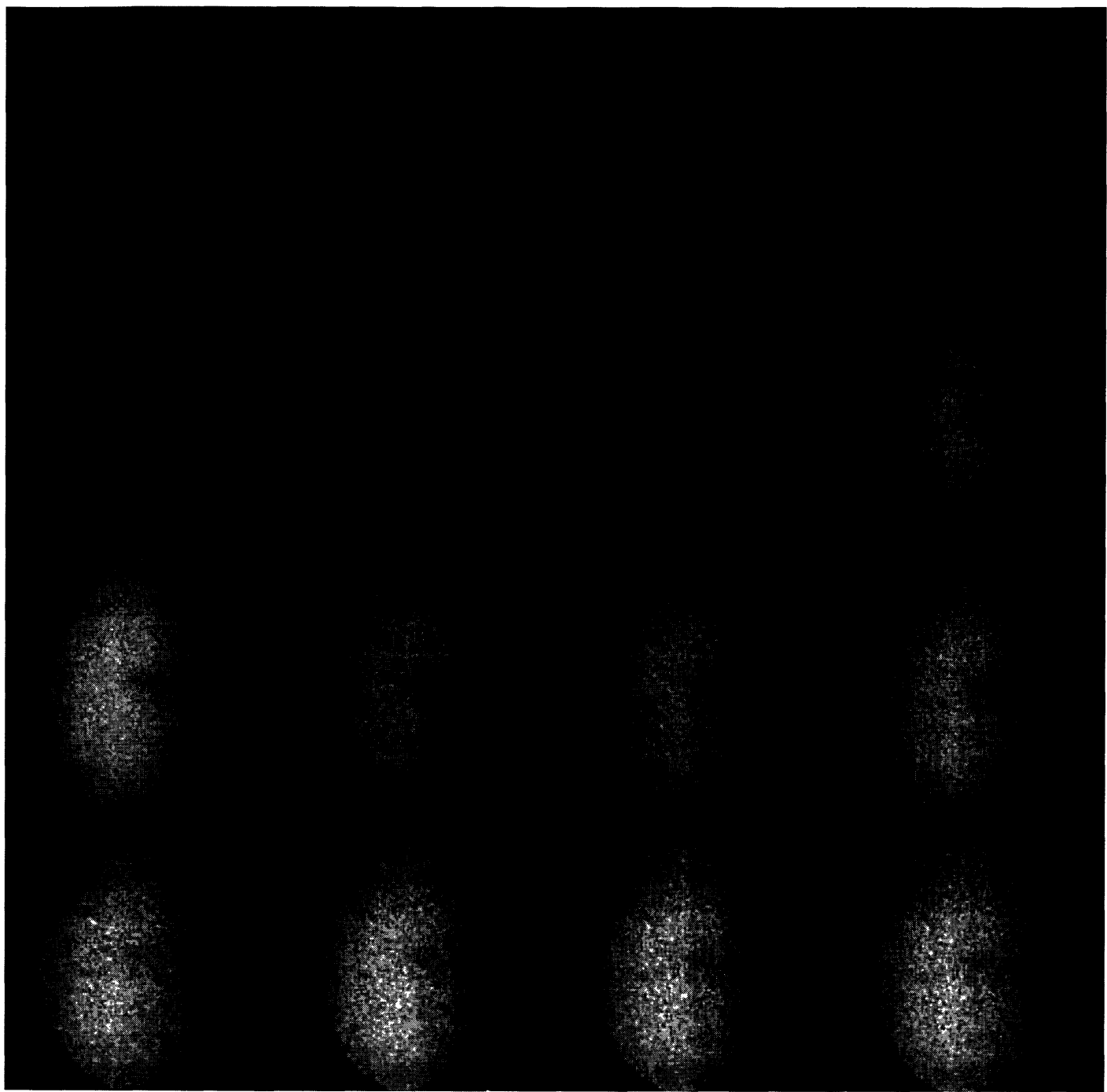

Fig. 5. - A 16 frame digital image of Nafion following background-stripping of the energy filtered image sequence recorded from $\Delta E=660 \mathrm{eV}$ (top left hand side) to $735 \mathrm{eV}$ (bottom right hand side) using the DT software. An image of the intermediate aperture in seen in each frame (diameter $400 \mathrm{~nm}$ ), but some artifactual distortion of each image is apparent following conversion from rectangular to square pixels for reproduction. The marked fluorine K-edge grey level intensity increase is seen in images 8-16.

Two practical constraints on image sequence analysis: Firstly, the steepening of a fitted polynomial curve from Nafion towards the lower ROI sizes suggested that there was a strong bias of energy loss information in the centre of the image. Pre-edge stripped images did not show any centre bias compared to post-edge images (see Fig. 5) and a profile of the electron beam viewed without a specimen showed a strong centre bias (data not shown). Since the distribution of fluorine in Nafion thin films was even, we conclude that the centre bias of intensity associated with the electron energy loss of fluorine in Nafion was simply a function of differential electron dose across the 
image field. Secondly, the characteristics of the grey level intensity modulations at the centre and the edge of images of Nafion assessed following collection of sequences using a $1 \mathrm{eV}, 2.5 \mathrm{eV}$ and 5 $\mathrm{eV}$ spectrometer exit slit also revealed the presence of a noticeable aberation-induced edge onset and maximum intensity shift by one frame $(5 \mathrm{eV})$ for regions of interest at the periphery of the field as compared to the same sized region at the centre of the field. If a slit width of $2.5-3 \mathrm{eV}$ or greater was used then no such $\mathrm{eV}$ shift was detectable. Whilst summing images over $30-45$ $\mathrm{eV}$ to maximise element sensitivity largely eliminated the effect of this phenomenon for semiquantitative analysis of image sequences, such aberation effects would become significant if the number of images collected for simple qualitative element distribution was restricted to only a few.

\section{Concentration-Dependent Binding of Titanium Dichloride to Purified RNA}

Figure 6 shows a representative stripped difference spectrum of MKT-4-incubated RNA following Image-EELS of a ROI comprising $100 \times 100 \mathrm{~nm}$, collected between $\Delta E=350$ and 670 $\mathrm{eV}$. Plotting the $\mathrm{Ti} / \mathrm{N}$ intensity ratio for the MKT-4-RNA conjugate against the concentration of MKT-4 during reaction by summing the intensity values associated with the nitrogen K-edge

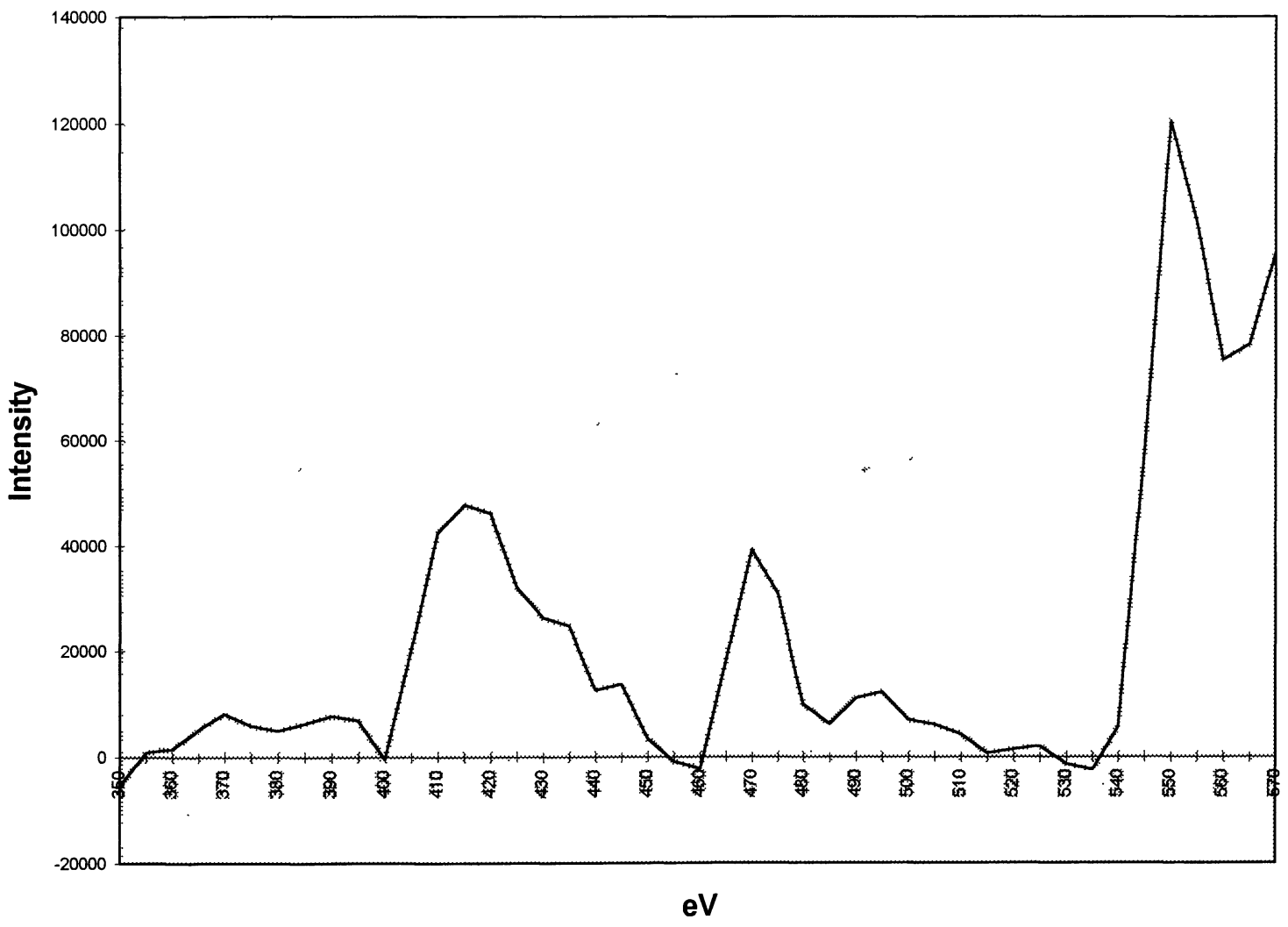

Fig. 6. - An image-EELS stripped intensity spectrum of the RNA-MKT-4 conjugate using the summed spectrum method. Well defined intensity increases associated with the nitrogen $\mathrm{K}$-edge at $\Delta E=400 \mathrm{eV}$, the titanium $L_{2,3}$-edge at $465 \mathrm{eV}$ and the oxygen $\mathrm{K}$-edge at $535 \mathrm{eV}$ form the basis of intensity ratio calculations. The calculated background is represented by 0 intensity. 
$(\Delta E=400-455 \mathrm{eV})$ and titanium $L_{2,3}$-edge $(\Delta E=460-515 \mathrm{eV})$ for six ROI's (about 8000 pixels in size) in three sequences for each incubation suggested a simple concentration-dependent reaction of the drug with $\mathrm{RNA}$. The $\mathrm{Ti} / \mathrm{N}$ intensity ratios for the conjugate were found to be 1.01 (SE \pm 0.27$), 0.77( \pm 0.1), 0.67( \pm 0.14), 0.42( \pm 0.09)$ and $0.16( \pm 0.06)$ for $16,8,4,2$ and $1 \mathrm{mM}$ MKT-4 respectively. The titanium intensity/pixel $(1.5 \mathrm{~nm})$ was more variable with values of 32.6 ( \pm 3.5$), 29.5( \pm 2.9), 29.1( \pm 1.1), 20.5( \pm 2.6)$ and $10.5( \pm 2.2)$ for $16,8,4,2$ and $1 \mathrm{mM}$ MKT4 respectively. Spectra for acurate qualitative and semi-quantitative analysis could be obtained from smaller ROI's $(20 \times 20 \mathrm{~nm})$ at all drug concentrations but only in the three higher drug concentration incubations. These data served as a comparative reference for the relative cellular concentration of titanium in A2780 spheroids.

\section{Intracellular Localisation of Titanium in the Outer Cells of A2780 Multicellular Spheroids}

Titanium was found to be concentrated as small $(5 \mathrm{~nm})$ particles in both small and large cytoplasmic vesicles in the outer cells of multicellular spheroids $(\mathrm{Ti} / \mathrm{N}$ ratio $=2.41 \pm 0 / 43)$ at levels equivalent to more than twice that associated with the highest MKT-4-RNA conjugate concentration $(16 \mathrm{mM})$. This represents a greater than 50 -fold increase in the relative amount of drug compared to the original culture medium. Titanium was found to be diffuse within the remainder of the cellular compartments and was not particulate following examination at 50,000 times nagnification. Expressed as a $\mathrm{Ti} / \mathrm{N}$ intensity ratio, the relative amount of titanium associated with the cytoplasmic domains was almost half that associated with nuclear domains $(0.58 \pm 0.07$ and $0.85 \pm 0.2$ respectively), and equivalent to $4 \mathrm{mM}$ and $8 \mathrm{mM}$ MKT-4-RNA respectively. These values represent an 8 and 16-fold relative increase in the drug concentration associated with cytoplasm and the nucleus respectively compared to the original culture medium. No titanium could be detected in the nucleolus. Since the N/N intensity ratio for nucleus/cytoplasm was $1.8( \pm 0.2)$, it is likely that the binding of MKT-4 to these regions was non-selective and simply dependent on the local density of macromolecules. Titanium was not detectable in the nucleus of the same cells using SEELS analysis.

\section{Discussion}

The image-EELS technique has clear advantages over SEELS in the analysis of biological and organic materials. The rapid acquisition time greatly limits any effects of beam damage to the specimen whilst at the same time providing extremely well spatially resolved energy loss information. Once post-edge images have had their background contributions removed (stripped) the grey level intensity values for each pixel can be summed, without any from of normalisation, to yield good qualitative elemental distribution images. Pixel grey level value addition over a limited post-edge eV range can provide semi-quantitative energy-loss information, similar to data derived from conventional SEELS analysis but with much greater sensitivity. The image-EELS method can give useful comparative data of multiple elements from within one field of interest, making it very attractive for the analysis of biological specimens. The least-squares model, although computationally intensive, gave a good background fit for boron, fluorine, nitrogen and titanium, on as few as seven pre-edge images, when compared to the generally accepted three-window method. However, the shape of the background energy contribution below $200 \mathrm{eV}$ and the consequent magnitude of the energy loss signal after using a small number of pre-edge images with a $5 \mathrm{eV}$ window was highly dependent upon choice of the pre-edge energy range, and was restricted by the dynamic range of the video camera. Although in principle the use of a 2 or $3 \mathrm{eV}$ window would greatly enhance the accuracy of the background model, it is likely that the time taken to 
record image sequences over a sufficiently wide spectral range to allow good comparisons of two or more elements to be made would severely reduce its practical application simply by increasing irradiation time.

The variation in the received electron dose across the field at comparatively low magnifications (20,000 times) makes lateral interpretation of semi-quantitative data difficult. This becomes particularly important when comparing the relative quantities of different elements within the same sample, and would demand that each ROI be at the same concentric position in each image within a sequence relative to the beam centre (usually the centre of the image). It might be possible to use relative energy loss grey level profiles to describe the radial dose variation of the field and to then use it to derive an accommodatory function for determining differences between the received dose of one pixel and another, perhaps in the same way that one can correct for image shading. Such variation, however, is unlikely to affect elemental intensity ratio calculations.

Aberration has the effect of displacing spectral information within a single field by 1 or $2 \mathrm{eV}$ for various points in a sequence recorded at 20,000 times magnification. The edge onset and energy loss peak for a certain element within a region at the centre of a field may appear in the next one or two following images within a region at the field edge. For most Image-EELS analyses in our own laboratory, however, a slit width of $5 \mathrm{eV}$ was employed to maximise the number of energy loss information slices that could be recorded within the relatively short time period in which no significant mass loss of the specimen took place, so the practical effects of aberration for this kind of analysis are likely to be negligible. If energy resolution is an important parameter, as it might if it was necessary to resolve the contribution of two significantly overlapping ionisation edges, then interpretation of the sequence data set must accommodate the resultant lateral energy shift across the field. Analysing only the centre portion of a spectroscopic image sequence [3] is one way around this constraint. Our approach sacrifices energy resolution for an increase in the size of the analysed area.

By integrating the grey level value for regions of interest as described here, it is likely that both qualitative local elemental distributions (elemental maps) and simple elemental intensity ratios can be obtained in organic materials for comparative analyses from a single data set. Using reduced electron doses would markedly reduce radiation damage to the specimen, but would also increase the detection limit proportionally. In the current report, the received dose was maximised for a short period by focusing the beam $\left(10^{-8} \mathrm{~A}\right)$ in order to maximise sensitivity of the energy loss information. Analysis of pixel-wise variations in mass density by recording image sequences in the low-loss region, data deconvolution to improve accuracy of the calculated spectra and exploration of the non-parametric treatment of data proposed by the use of the correspondence analysis [6] will be used to refine further the methods described in this report.

In common with previous studies on the biodistribution of titanium in mice and human tumor cells following treatment in vivo [5,6], we found that the drug was concentrated in secondary lysosome-like vesicles as small particles following treatment of model micrometastatic tumors in vitro. More importantly, however, we found that there was a direct relationship between the amount of bound drug and the amount of nitrogen associated with intracellular domains using the intensity ratio of titanium/nitrogen as an indicator of the relative intracellular distribution of MKT-4. This amounted to an 8 and 16-fold accumulation of titanium within cytoplasmic and nuclear regions. Further analysis of this compound within cytotoxic drug-sensitive and resistant cell lines is underway and is likely to provide information leading to a greater understanding of the mechanisms of drug resistance. 


\section{Conclusions}

We characterise a method of extracting energy loss intensity information from energy filtered image sequences for the semi-quantitative analysis of boron and fluorine-containing polymers and for titanium-bound organic macromolecules using the integration of grey level values for pixels in combination with computer software written specifically for a Windows environment. We have shown that energy loss intensity information derived by this Image- EELS approach is considerably more sensitive than for a SEELS analysis and can be applied to an examination of the relative intracellular distribution of a titanium-containing cytotoxic drugs in experimental tumors.

\section{Acknowledgements}

We would like to thank the Scottish Hospital Endowments Research Trust and the Cancer Research Campaign for financial support. We would also like to thank Dr. Ze'eV Porat for practical advice on the preparation of Nafion and Kim Wilson for technical support.

\section{References}

[1] Hunt J.A. and Williams D.B., Ultramicroscopy 3 (1991) 147.

[2] Lavergne J.L., Foa C., Bongrand P., Seux D. and Martin J.M., J. Microsc. 174 (1994) 196.

[3] Kortje K.H., J. Microsc. 174 (1994) 149.

[4] Jeanguillaume C., Trebia P. and Colliex C., Ultramicroscopy 32 (1978) 12.

[5] Kopf-Maier P. and Martin R., Virchows Archiv B Cell Pathol 57 (1989) 213.

[6] Kopf-Maier P., J. Structural Biol. 105 (1990) 35.

[7] Sutherland R.M., Science 240 (1989) 177.

[8] Gaze M.N., Huxham I.M., Mairs R.J. and Barret A., Int. J. Cancer 47 (1991) 875.

[9] Egerton R.F., Electron Energy Loss Spectroscopy in the Electron Microscope (Plenum Press, New York \& London, 1986).

[10] DeBruijn W.C., Sorber C.W.J., Gelsema E.S., Beckers A.L.D. and Jongkind J.F., Scanning Microsc. 7 (1993) 693.

[11] Beckers A.L.D., Gelsema E.S.,DeBruijn W.C. , J Microsc. 171 (1993) 87.

[12] Gelsema E.S., Beckers A.L.D., Sorber C.W.J. and DeBruijn W.C., J. Microsc. 166 (1992) 287. 